\title{
JSP Special Issue on Information Processing in Living Systems
}

\author{
Thierry Mora ${ }^{1} \cdot$ Luca Peliti $^{2}$ (D) Olivier Rivoire ${ }^{3}$
}

Received: 12 January 2016 / Accepted: 21 January 2016 / Published online: 9 February 2016

(C) Springer Science+Business Media New York 2016

Living systems are information-processing systems: they need to copy internal information, e.g., contained in their DNA, for producing their proteins - and regulating their productionor for reproducing. They also need to monitor their environment and their internal state, and to control their activity based on the collected informations. All this using molecular machinery, continually subject to thermal noise. Trying to understand how living systems manage these tasks defines an area of questions at the cross-roads between statistical physics, information theory and biology. The contributions contained in the present Special Issue of the Journal of Statistical Physics, which cover a wide-range of topics from information-dissipation tradeoffs to statistical inference and issues of biological noise, hope to shed some light on these questions.

It has been known for quite some time that information processing requires thermodynamical dissipation. A number of contributions address the question of how living systems handle dissipation-information tradeoffs in information transmission and in sensing. Mancini, Marsili and Walczak consider biochemical regulatory circuits in different regimes, eventually asking how to design a signaling network to optimize function in the worst possible environment. They identify a universal motif that is optimal at large dissipation. François and Altan-Bonnet ask how cells are able to take decision based on the quality of surrounding ligands, almost irrespective of their quantity. They suggest a mechanism based on adaptive sorting, and show how kinetic proofreading with negative feedback can implement adaptive sorting in the immune system. Mehta, Lang and Schwab detail, using several simple

\footnotetext{
$凶 \quad$ Luca Peliti

luca@peliti.org

Thierry Mora

tmora@lps.ens.fr

Olivier Rivoire

olivier.rivoire@ujf-grenoble.fr

1 Laboratoire de Physique Statistique, Ecole Normale Supérieure, 75005 Paris, France

2 Simons Center for Systems Biology, Institute for Advanced Study, Princeton, NJ 08540, USA

3 Laboratoire Interdisciplinaire de Physique, Université de Grenoble-Alpes, 38000 Grenoble, France
} 
examples, how energy consumption in cellular circuits allow for reaching increased specificity, manipulating dynamics, reducing variability, amplifying signals and erasing memory, and discuss the implications of these ideas to synthetic biology. Pigolotti and Sartori focus on information-dissipation tradeoffs in template-assisted polymerization, considering several possible designs of proofreading protocols, and finding that only a limited number of protocols lead to improved accuracy. Murugan and Vaikuntanathan generalize the notion of proofreading by exploiting the idea of dynamical phases-typical trajectories executed by non-equilibrium systems. Functions like error correction, ultra-sensitivity, precise adaptation and accurate concentration measurement can be achieved by switching between different dynamical phases via a rather small control on the dynamics. These works show how living systems perform these information-processing tasks, at the molecular level, by a number of Maxwell-Demon-like mechanisms whose functioning can be understood by the tools of non-equilibrium statistical mechanics and of stochastic thermodynamics.

Inference problems are another point of convergence between information theory, statistical mechanics and biological problems. This convergence is now driven by large-scale quantitative data in several areas of biology, particularly in genomics with the sequencing of DNA sequences and neurobiology with the recordings of neuronal activities. Central to applications of ideas from information theory and statistical mechanics is the concept of entropy, along with its many derivatives (mutual information, Kullback-Leibler divergence, ...). In this issue, Atwal and Kinney thus show how inference methods based on mutual information can overcome the shortcomings of more standard approaches when inferring the function of biological sequences from the output of massively parallel experiments. Similarly, Kappen and Ruiz apply ideas from cross-entropy method to devise efficient computational schemes for solving stochastic control problems - that are formally equivalent to high-dimensional inference problems encountered in neuroscience. Barton and collaborators present different calculations of the entropy of a protein family, i.e., of a set of protein sequences sharing a similar function; in particular, they show how to go beyond estimations based on standard hidden-Markov chains by considering maximum-entropy distributions, which map the problem to Potts models with pairwise interactions. The principle of maximum entropy has now been applied to several biological datasets, with the consistent observation that accounting only for pairwise correlations is sufficient to obtain a good approximate description. Merchan and Nemenman examine the origin of this observation by analyzing several toy models with high-order interactions and come to the conclusion that densely connected systems are generically well-described by pairwise models. In this problem as in others, understanding why an inference method works well, and therefore understanding what inference method is best suited, ultimately boils down to the problem of understanding how and why Nature has designed biological systems in specific ways. Information processing is at the heart of this question, as natural selection often acts to favor biological designs that best process and encode information. Salisbury and Palmer thus discuss different ways in which the retina may be considered as an efficient encoder of visual signals. On a different scale, Rivoire presents models to analyze how a population of reproducing individuals best process informations from past generations and from the current environment. Remarkably, the two studies come to the same conclusion: optimal information processing in biology has to be judged in light of the statistics of environments under which the systems evolved.

Biological systems communicate and process information through biochemical signaling. The accuracy of this communication is physically limited by noise arising from the small number of molecules involved in information transmission. The consequences of this physical limit on the accuracy of concentration sensing by biological units, and in particular by receptors expressed at the surface of cells, was first investigated in the pioneering work of 
Berg and Purcell in 1977. Past work has demonstrated that many organisms indeed operate close to this physical limit. In this issue, Endres, Wingreen and Aquino revisit the bound derived by Berg and Purcell for a single receptor by reviewing more general schemes for concentration readout, as well as the effect of ligand rebinding. Bo and Celani investigate the detection of concentration changes by cooperative receptors. Gene regulation can also be viewed as an example of information transmission. Specific binding sites on the DNA act as receptors for the transcription factors that regulate downstream genes. In this case, the problem of concentration sensing is complicated by the need to discriminate between a large number of potential binding sites. Samir, Kagan and Tlusty investigate the trade-off between the conflicting requirements of discrimination power and quick information transfer. Biochemical noise is not limited to receptors. It also affects downstream chemical reactions, which also rely on small number of molecules. Ten Wolde et al. review the filtering of receptor noise by these signaling networks, and discuss the energetic costs associated with optimal performance. Kobayashi, Yokota and Aihara study the propagation of biochemical noise in networks with feedback loops and the role of these loops on network efficiency.

We hope that this issue will contribute to the convergence of concepts and methods in studies of information processing in biological processes and statistical physics. 